\title{
LA EDUCACIÓN SUPERIOR TRANSNACIONAL EN MÉXICO: EL CASO SYLVAN-UNIVERSIDAD DEL VALLE DE MÉXICO
}

\author{
ROBERTO RODRÍGUEZ GÓMEZ*
}

\begin{abstract}
RESUMEN: En el marco de apertura al comercio internacional que han desarrollado los últimos gobiernos de México ¿cuáles han sido las implicaciones para la educación superior? La presente contribución se ocupa del tema a través de una revisión general del proceso de apertura comercial, el análisis de los datos sobre inversión extranjera directa en México en el sector de educación privada, y mediante el estudio de un caso: la presencia del consorcio Sylvan Learning Systems en la Universidad del Valle de México, institución privada que ofrece educación de bachillerato, licenciatura y postgrado. El artículo se divide en cinco secciones. La primera examina la postura del gobierno mexicano ante los procesos de globalización y regionalización del comercio internacional. La segunda trata de la postura mexicana ante la perspectiva de la transnacionalización educativa. La tercera trata del marco legal mexicano para la inversión extranjera directa en educación. La cuarta presenta datos acerca del volumen de inversión extranjera directa en el sector educativo mexicano. La quinta contiene el estudio de caso.
\end{abstract}

Palabras claves: Transnacionalización universitaria. Inversión extranjera directa en educación superior. Educación superior privada en México.

Transnational higher education in Mexico: The Sylvan-Universidad Del Valle de Mexico case

ABSTRACT: This paper discusses the impact of transnationalisation on Mexican higher education system in four steps: First, it examines the Mexican government position about the global international trade. Second, it exposes the general Mexican position about the

* Universidad Nacional Autónoma de México (UNAM), Centro de Estudios sobre la Universidad, Seminario de Educación Superior. E-mail: roberto@servidor.unam.mx 
educative transnationalisation. Third, it refers the Mexican legal frame about direct foreign investment in education. Fourth, it displays data about the volume of direct foreign investment in the Mexican educative sector. Finally, the attention turns to a specific case of transnationalisation: the presence of a partnership between Sylvan Learning Systems and the University of the Valley of Mexico, private institution that offers education of baccalaureate, degree and postdegree.

Key words: University transnationalization. Direct foreign intervention in higher education. Private higher education in Mexico.

\section{El gobierno de México ante los procesos de globalización y regionalización del comercio internacional}

\section{7 partir del gobierno de Miguel de la Madrid, se inició en Méxi- co un viraje de política económica consistente en la apertura al comercio internacional y la atracción de inversión extranjera di-} recta (IED) en la mayor parte de los sectores económicos del país. En noviembre de 1986 se cumplió la incorporación formal de México al Acuerdo General de Tarifas y Aranceles (GATT), antecedente de la actual Organización Mundial del Comercio (OMC). En el periodo presidencial de Carlos Salinas de Gortari se desarrolló y concretó el Tratado de Libre Comercio entre México, Estados Unidos y Canadá (TLCAN). El tratado norteamericano se negoció en 1991 y 1992 y entró en vigor el 1ro. de enero de 1994. En 1993 México se incorporó al Mecanismo de Cooperación Asia Pacífico (APEC) y en mayo de 1994 se aceptó al país como miembro 25 de la Organización para Cooperación y Desarrollo Económico (OCDE). Durante los gobiernos de Ernesto Zedillo y Vicente Fox, continuó la firma de tratados de libre comercio de carácter bilateral y multilateral. ${ }^{1}$

México es el país que ha firmado el mayor número de tratados de libre comercio en el mundo, lo que demuestra claramente el interés gubernamental en el modelo económico basado en la apertura comercial. Como parte de la misma política, el gobierno ha cumplido y sostiene un papel activo en los foros y mecanismos encargados de procesar la liberalización y desregulación del libre comercio internacional a escala global y regional. En el primer caso, a través de la OMC, y en el segundo, que atañe al ámbito hemisférico en que se incluye al país, mediante el Área de Libre Comercio de las Américas (ALCA). Además, el actual gobierno pro- 
La educación superior transnacional en México...

puso un mecanismo complementario a los ya indicados: el Plan Puebla Panamá (PPP), que incluye acuerdos de intercambio en la región mesosamericana. ${ }^{2}$ El PPP se lanzó el 15 de junio de 2001, con el apoyo del Banco Interamericano de Desarrollo, como un esquema regional que integraría proyectos de colaboración en distintas áreas de comercio, desarrollo de infraestructura económica y desarrollo social. ${ }^{3}$

Las implicaciones para el sector educativo de la presencia de México en los procesos de globalización y regionalización, así como en los acuerdos de cooperación derivados, pueden situarse principalmente en dos niveles. En primer lugar, en el que corresponde a las repercusiones normativas y sustantivas derivadas de la firma de acuerdos de colaboración internacional que comprenden al sector educativo en sus distintos niveles y modalidades y, en segundo lugar, en el plano que atañe a la liberalización del comercio en el sector de la educación privada. Para enfocar nuestro tema, veamos a continuación cómo está siendo procesada, en el plano global, la apertura comercial del sector educativo, y dentro de este proceso cuál es la postura de México.

2. La liberalización del comercio internacional en el sector de educación privada: aspectos generales y postura de México

Al término de la Ronda Uruguay del Acuerdo General de Tarifas y Aranceles (GATT) en 1995, se consideró agotada la estructura del Acuerdo y se decidió reemplazarla por un organismo: la Organización Mundial del Comercio (OMC). Desde su comienzo, la OMC incluyó el capítulo del GATT sobre comercio de servicios, conocido como Acuerdo General para el Comercio de Servicios o GATS. Los servicios considerados son: negocios, comunicación, construcción e ingeniería, cultura, distribución, educación, medio ambiente, salud, servicios financieros, recreación, transportes, y turismo. Cada uno de los sectores se desglosa en subsectores y actividades hasta abarcar aproximadamente 160 rubros. Para el sector educativo se consideran cuatro categorías: servicios de enseñanza primaria, servicios de enseñanza secundaria, servicios de enseñanza superior y servicios de enseñanza para adultos, además se especifica el tipo "otros servicios de enseñanza" que incluye cualquier modalidad fuera del esquema general.

Para cada servicio se consideran cuatro modos de suministro: a) suministro transfronterizo: el proveedor exporta el servicio desde su país 
de origen; b) consumo en el extranjero: el demandante acude al extranjero a consumir el servicio; c) presencia comercial: el proveedor instala en el extranjero el servicio; y d) presencia de personas naturales que suministran el servicio en el extranjero. Una vez que un país conviene en participar en el GATS queda sujeto a las obligaciones generales del Acuerdo, establece compromisos generales de acceso a sus mercados $\mathrm{y}$ de trato nacional en sectores y subsectores particulares, y declara o recibe intereses específicos de negocios con países miembros de la organización.

La negociación de servicios bajo las reglas del GATS se inició a comienzos del 2000, bajo la supervisión del Consejo para el Comercio de Servicios de la OMC. En marzo de 2001 el Consejo completó un esquema general para guiar las negociaciones. La Declaración de Doha del 14 de noviembre de 2001 convalidó el trabajo realizado por el Consejo y estableció un calendario para organizar las negociaciones en el marco del Acuerdo. En éste las fechas claves son: solicitudes de acceso a mercados (junio 2002); ofertas iniciales de acceso a mercados (marzo 2003); revisión de resultados alcanzados (septiembre 10-14 en la 5ta. Conferencia Ministerial de la OMC. El límite para la emisión del Acuerdo es el 1 ro. de enero de 2005.

Las obligaciones generales de los suscriptores del GATS son cuatro y aluden al compromiso de cada país signatario del Acuerdo de ofrecer a los demás: a) trato de nación más favorecida, que implica extender a todos los países las condiciones más favorables convenidas con cualquier país o conjunto de ellos; b) transparencia, que se traduce en ofrecer libre acceso a la información relevante para efectos comerciales; c) trato nacional, que significa tratar a los proveedores extranjeros de manera igual que a los nacionales, salvo reservas pactadas por caso; y d) acceso a los mercados.

Además de esas obligaciones, cada gobierno identifica sus condiciones en un Protocolo de Compromisos Específicos (Schedule of Specific Commitments) en que se describe, para cada sector, los subsectores en que se ofrecen condiciones de trato nacional o de libre acceso a mercado. La lista incluye también los subsectores de acceso limitado, indicando en cada caso el tipo de restricción que opera.

México presentó al GATT su Protocolo el 14 de abril de 1994 (GATS/sc/56). El contenido del documento replica, fundamentalmente, las condiciones de apertura para la inversión en servicios previstas en el 
La educación superior transnacional en México...

TLCAN, así como las limitaciones definidas en el acuerdo trilateral, de las que nos ocuparemos más adelante.

En las negociaciones generales del GATS hasta la fecha, los servicios de enseñanza son el sector que ha sido objeto de menos compromisos, después de los servicios de energía. Únicamente 30 de los 144 países signatarios, entre ellos México, han enviado la descripción de los servicios educativos susceptibles de incorporar en el Acuerdo, así como las condiciones a las que sujetan su participación. Además, sólo cuatro han definido posturas generales, todas ellas a favor de la apertura comercial en educación (Japón, Australia, Nueva Zelanda y Estados Unidos). La propuesta estadounidense recomienda a "los Miembros de la OMC que todavía no hayan contraído compromisos sobre los servicios de enseñanza superior, enseñanza para adultos y capacitación formulen sus compromisos". "Además, se recomienda incluir dos rubros adicionales en la clasificación de servicios educativos: los servicios de capacitación y los servicios de pruebas educativas.

En otras partes del mundo la academia ha reaccionado con prontitud contra la incorporación de la educación superior en el GATS. Es el caso de las conferencias de rectores de Europa, de la Asociación Europea de Universidades y de los Consejos de Acreditación de Europa, Estados Unidos y Canadá. En una declaración conjunta afirman enfáticamente: "Nuestros respectivos países deben evitar compromisos en materia de Servicios de Educación Superior o en categorías relacionadas de Educación de Adultos y otros Servicios Educativos en el contexto del GATS".

La postura de México ante el GATS, y en consecuencia en las negociaciones del ALCA, se deriva del enfoque general, compromisos y limitaciones establecidos en el TLCAN y en resto de los tratados de libre comercio suscritos hasta la fecha. En general, esta postura considera la apertura del todo el segmento educación privada (de preescolar a postgrado en cualquier combinación y modalidad), salvo las limitaciones que provienen de la normativa vigente. A continuación pasaremos somera revista a ese marco normativo.

\section{El marco legal de la inversión extranjera directa en educación}

El marco legal vigente proviene de varias regulaciones: Ley de Inversiones Extranjeras (1993), Ley General de Educación (1993) y nor- 
mas secundarias del sector, Tratado de Libre Comercio con EU y Canadá (2994) y posteriores tratados de libre comercio de carácter bilateral o multilateral. En la primera se asegura la posibilidad de inversión extranjera en todo el sector de educación privada, incluida la educación superior y los servicios mixtos, es decir la provisión educativa en cualquier combinación de niveles y modalidades. El artículo 8 de la Ley de Inversiones Extranjeras indica que "se requiere resolución favorable de la Comisión (de inversiones extranjeras) para que la inversión extranjera participe en un porcentaje mayor al $49 \%$ en las actividades económicas y sociedades que se mencionan a continuación (...)". La lista de actividades incluye, entre otros, "los servicios privados de educación preescolar, primaria, secundaria, media superior, superior y combinados".

Por su parte, la Ley General de Educación establece la obligación de los particulares que imparten educación superior a obtener, ante autoridad competente, un reconocimiento de validez oficial para cada plan de estudios (Art. 55). En lo que atañe al sector de educación superior privada es aplicable el Acuerdo 279 de la SEP que norma el Registro de Validez Oficial de Estudios (RVOE), ${ }^{6}$ así como los Acuerdos $286,{ }^{7}$ que se refiere al procedimiento de revalidación de estudios realizados en el extranjero, y $328^{8}$ que modifica en algunos aspectos el Acuerdo 286 previo. La normativa secundaria establece, por un lado, cuáles son las autoridades educativas que pueden autorizar programas de educación superior y cuáles son los requisitos a cumplir para obtener registro (Acuerdo 279). Por otro, se establece el procedimiento de revalidación, mediante tablas de equivalencia y otros mecanismos, de estudios efectuados en el extranjero que, como ya se indicó, corresponden a una de las formas de comercio internacional (consumo en el extranjero).

En octubre del año 2000 se estableció el Consejo Nacional de Acreditación de la Educación Superior A.C. (COPAES) como la instancia "reconocida por el Gobierno Federal, a través de la SEP, para conferir reconocimiento formal a organizaciones cuyo fin sea acreditar programas académicos de educación superior que ofrezcan instituciones públicas y particulares, previa valoración, entre otros aspectos, de sus procedimientos y de su imparcialidad".?

Finalmente, tanto el TLCAN como otros acuerdos de libre comercio en que participa México reflejan la normatividad citada. En el tex- 
La educación superior transnacional en México...

to del TLCAN se incluye la norma de inversión extranjera en materia educativa (Anexo 1 de la Lista México), y la disposición que obliga a tramitar autorización oficial (Anexo 5 de la Lista México).

En consecuencia, la postura de México ante la importación-exportación de servicios de educación superior privada es que se acepta la presencia de proveedores privados extranjeros siempre y cuando se sujeten a las normas de autorización de carácter comercial (regulación de inversiones extranjeras) y educativo (regulaciones para la educación superior privada). A continuación observaremos cuál a sido el comportamiento de la inversión extranjera directa en el sector de educación privada.

\section{La inversión extranjera directa en la educación privada en México 1994-2003}

Como se indica en la Tabla 1, el monto total acumulado de inversión extranjera directa (IED) en servicios educativos prestados por el sector privado, en el período 1994 a 2003, asciende a un total cercano a 40 millones de dólares. Casi el 90 por ciento de toda la inversión en el período está situada en el año 2000 y es explicable por una sola operación, la adquisición de la mayor parte de la Universidad del Valle de México por el consorcio Sylvan Learning Systems, de la que nos ocuparemos más adelante.

El resto de las operaciones del periodo se explica por inversiones de mucho menor cuantía, en rubros tales como enseñanza de idiomas (2,6 millones de dólares), servicios de educación especial (2,3 millones de dólares) y educación superior (1,7 millones de dólares). Considérese que el periodo reportado se inicia con la puesta en vigencia de la Ley de Inversiones Extranjeras de 1993 y el inicio del TLCAN.

La Tabla 2 ofrece información sobre el número de empresas que registran IED en sus operaciones. Se trata, en total, de 113 empresas en el periodo 1994-2003, la mayor parte de las cuales (54 por ciento) está concentrada en la enseñanza de idiomas. En lo que respecta a la educación superior, únicamente se consignan siete instituciones con presencia de IED, aunque con seguridad se añaden otras entre las 14 instituciones que cuentan con servicios combinados (varios niveles de enseñanza). Aún así, un máximo de 21 instituciones con educación superior habrían con- 


\section{Tabla 1}

México: Inversión extranjera directa en servicios educativos prestados por el sector privado (En US dólares)

\begin{tabular}{|l|r|}
\hline \multicolumn{1}{|c|}{ Año } & \multicolumn{1}{|c|}{ Total } \\
\hline 1994 & $168,267.7$ \\
\hline 1995 & $92,113.8$ \\
\hline 1996 & $826,532.9$ \\
\hline 1997 & $649,663.6$ \\
\hline 1998 & $389,509.8$ \\
\hline 1999 & $2,700,020.9$ \\
\hline 2000 & $34,337,805.7$ \\
\hline 2001 & $789,185.1$ \\
\hline 2002 & $-1,370,345.3$ \\
\hline $2003 *$ & $212,529.1$ \\
\hline Acumulado 1994-2003 & $38,795,283.3$ \\
\hline
\end{tabular}

Fuente: Secretaría de Economía. Dirección General de Inversión Extranjera.* Comprende hasta 30 de junio de 2003.

tado con IED para sus operaciones. Debido a la normativa en la materia, es poco probable que exista un solo caso en que la IED se haya concretado en el establecimiento de una universidad u otra modalidad de educación superior sin presencia de capital nacional. No se cuenta con información específica sobre la proporción, en cada caso, de la IED en las sociedades nacionales que hayan obtenido recursos por esta vía.

Lo más significativo de la información sobre el monto y número de operaciones vinculadas al capital extranjero en el campo de los servicios de educación superior es la escasa presencia de inversionistas considerando tanto el periodo de tiempo (casi una década) como el grado de apertura prevaleciente. En 2000, año "pico" de la serie estadística, la IED en México fue de un total de 16,448.7 millones de dólares (Secretaría de Economía); en ese total, la IED en educación representa el 0,2 por ciento. Si se contrastan estas cifras con las de otros sectores de servicios, por ejemplo finanzas (que en el lapso 1999-2003 recogió más del 30 por ciento de toda la IED en México), turismo (3,5 por ciento 
La educación superior transnacional en México...

\section{Tabla 2}

Empresas con inversión extranjera directa en servicios educativos privados (1994-2003)

\begin{tabular}{|l|c|c|}
\hline Actividad & Empresas & Porcentaje \\
\hline Total & 113 & 100,0 \\
\hline 921107 Servicios de enseñanza comercial y de idiomas. & 61 & 54,0 \\
\hline $\begin{array}{l}\text { 921106 Servicios privados de educación que combinan los niveles } \\
\text { de enseñanza preescolar, primaria, secundaria, media superior y superior. }\end{array}$ & 14 & 12,4 \\
\hline 921111 Servicios privados de educación especial. & 14 & 12,4 \\
\hline $\begin{array}{l}\text { 921109 Servicios de enseñanza de música, danza } \\
\text { y otros servicios particulares de enseñanza. }\end{array}$ & 7 & 6,2 \\
\hline 921105 Servicios privados de educación superior. & 7 & 6,2 \\
\hline 921108 Servicios de capacitación técnica, de oficios y artesanías. & 6 & 5,3 \\
\hline 921110 Servicios de educación por parte de profesores particulares. & 3 & 2,7 \\
\hline 921101 Servicios privados de educación preescolar. & 1 & 0,9 \\
\hline
\end{tabular}

Fuente: Secretaría de Economía. Dirección General de Inversión Extranjera. Nota: Comprende hasta 30 de junio de 2003.

de la IED total) o telecomunicaciones y transporte (2,5 por ciento de la IED total), se aprecia con facilidad el relativamente corto interés, hasta el momento, de los inversionistas en el segmento educativo.

La explicación del fenómeno debe considerar que, a diferencia de otros sectores de servicios, en el educativo existe una provisión estatal mayoritaria, un ritmo de privatización lento y muy concentrado en el segmento superior, y un mercado potencial acotado y competido por múltiples proveedores locales, algunos de ellos con estándares de calidad apreciables. Hay sin embargo una excepción a la pauta general, cuyas características y desarrollo obligan a matizar el corolario anterior. Con ese propósito, a continuación se presenta el caso de la inversión de Sylvan Learning Systems en la Universidad del Valle de México.

\section{Sylvan Learning Systems Inc. y la Universidad del Valle de México}

Para presentar el caso de estudio nos concentraremos en dos aspectos. En primer lugar, el desarrollo reciente del consorcio Sylvan, 
destacando dos fases que describen la dinámica empresarial del mismo. La primera puede ser descrita como una etapa de diversificación y experimentación de ofertas en servicios educativos, mientras que la segunda se aprecia como una etapa de concentración y definición en el terreno de la educación superior, a través del desarrollo de una red internacional de universidades, en que se incluye a la Universidad del Valle de México (UVM). En segundo lugar, nos ocuparemos de la relación entre el consorcio y la UVM destacando los cambios perceptibles en esa organización a partir de la inversión extranjera en ella.

\section{a) Desarrollo reciente del consorcio Sylvan}

Uno de los participantes más activos en el contexto de la educación superior transnacional es el consorcio Sylvan. En su origen, la firma estaba dedicada al diseño de servicios de capacitación, entrenamiento y educación formal mediante contratos con instituciones y empresas (Sylvan Education Solutions). Posteriormente se ocupó del desarrollo de servicios de regularización y preparación de exámenes a través de los Centros de Aprendizaje Sylvan, fundados en 1979 e instalados en EUA, Canadá y Europa. Más adelante, en 1997, se adquirieron los Institutos Wall Street (WSI) de enseñanza de inglés y se buscó su expansión internacional a través de la venta de franquicias. Al presente los WSI suman más de 350 establecimientos en 26 países, con una inscripción de más de 140 mil estudiantes.

En 1999, Sylvan compró la mayor parte de la Universidad Europea de Madrid, institución establecida como universidad privada en 1995 a partir del Centro Europeo de Estudios Superiores. Con esa operación, el consorcio dio inicio a la integración de una red de instituciones de enseñanza superior que se ha extendido mediante la compra total o parcial de varias escuelas y universidades en EU y en países de Europa, América Latina y Asia. En la actualidad, la red cuenta con cuatro instituciones de educación superior a distancia, nueve instituciones universitarias de enseñanza presencial, y una matrícula total de aproximadamente 120 mil estudiantes.

El crecimiento de las actividades relacionadas con la educación superior, principalmente el despliegue de la red universitaria, llevó al consorcio Sylvan a la decisión de desprenderse del resto de sus compañías de servicios. En junio de 2003 se cumplió la venta de Sylvan 
La educación superior transnacional en México...

Learning Center, Schulerhilfe y Sylvan Education Solutions, así como de la proporción de acciones de e-Sylvan y Connections Academy en manos del consorcio. El bloque fue adquirido por una compañía formada para tal propósito, la Educate Operating Company (Educate Inc.), establecida, al igual que Sylvan, en Baltimore, EUA. Además se determinó la liquidación de los wsi con la expectativa de finiquitar la operación en 2004. Por su parte, el segmento encargado de la red universitaria cambió oficialmente su denominación de Sylvan Learning Systems Inc. a Laureate Education Inc. a partir del 17 de mayo de 2004.

Como ya se indicó, la red universitaria consta de modalidades en línea y tradicionales. Las primeras incluyen las siguientes instituciones: Carter \& Associates (EUA), dedicada principalmente a la actualización de profesores en servicio; National Technological University (EUA), que ofrece cursos de grado y postgrado en disciplinas tecnológicas en asociación con varias universidades norteamericanas; Walden University, institución pionera del modelo de universidad virtual; y K.I.T. Learning, institución holandesa que ofrece cursos de tecnológicas informáticas a través de la Universidad de Liverpool (Inglaterra).

Las escuelas y universidades adscritas a la red en la modalidad presencial son, en orden de adquisición: Universidad Europea de Madrid (España), Les Roches Hotel Management School (Suiza, España y China), Ecole Supérieure du Commerce Extérieur (Francia), Universidad de las Américas (Chile y Ecuador), Universidad del Valle de México (México), Glion Institute of Higher Education (Suiza), Academia de Idiomas y Estudios Profesionales (Chile), Universidad Andrés Bello (Chile), Universidad Interamericana (Costa Rica y Panamá). ${ }^{10}$

Las decisiones de compra o asociación de instituciones por parte de Sylvan se basan en criterios tales como el nivel de consolidación académico logrado, el potencial de crecimiento local y regional, la existencia de vínculos y relaciones con otros sectores, así como las posibilidades de participación en la red universitaria. En general, las unidades participantes mantienen sus esquemas curriculares originales, aunque se responsabilizan de desarrollar los criterios del "sello Sylvan", a saber: formación orientada al empleo, conocimiento de inglés e informática. Aunado a lo anterior, las instituciones se comprometen a apoyar a los estudiantes en la búsqueda de colocación profesional, así como brindar opciones de movilidad internacional en el marco de la red. Asimismo, varias de las escuelas y universidades afiliadas cuentan o están proce- 


\section{Tabla 3}

\section{Red Internacional de Universidades Sylvan (Laureate Inc.)}

\begin{tabular}{|c|c|}
\hline Educación superior a distancia ( $w$ eb based) & Educación superior presencial (campus based) \\
\hline $\begin{array}{l}\text { Canter \& Associates (EUA) } \\
\text { Adquirida en 1998. Propiedad completa. } \\
\text { Aproximadamente } 4.500 \text { estudiantes. }\end{array}$ & $\begin{array}{l}\text { Universidad Europea de Madrid (España) } \\
\text { Adquirida en 1999. } 78 \% \text { de la propiedad. } \\
\text { Aproximadamente } 8.000 \text { estudiantes. }\end{array}$ \\
\hline $\begin{array}{l}\text { National Technological University (EUA). } \\
\text { Adqurida en 2002. Propiedad completa. } \\
\text { Aproximadamente } 2.000 \text { estudiantes }\end{array}$ & $\begin{array}{l}\text { Les Roches Hotel Man agem ent School (Suiza, España y China) } \\
\text { Adquirida en 2000. Propiedad completa. } \\
\text { Filial en Marbella. En } 2004 \text { inicia una filial en Shanghai. } \\
\text { Aproximadamente } 800 \text { estudiantes. }\end{array}$ \\
\hline $\begin{array}{l}\text { Walden University (EUA) } \\
\text { Adquirida en 2001. } 51 \% \text { de la propiedad. } \\
\text { Aproximadamente } 1.600 \text { estudiantes. }\end{array}$ & $\begin{array}{l}\text { Universidad de las Américas (Chile y Ecuador) } \\
\text { Adquirida en } 2000 . \text { Propiedad parcial. } \\
\text { Aproximadamente } 17.500 \text { estudiantes (Chile). }\end{array}$ \\
\hline \multirow[t]{6}{*}{$\begin{array}{l}\text { K.I.T. Learning (Holanda) } \\
\text { Adquirida en } 2004 \text {. Propiedad completa. } \\
\text { Aproximadamente } 1.500 \text { estudiantes. }\end{array}$} & $\begin{array}{l}\text { Universidad del Valle de México (México) } \\
\text { Adquirida en } 2000.80 \% \text { de la propiedad. } \\
\text { Aproximadamente } 43.000 \text { estudiantes en } 18 \text { campus. }\end{array}$ \\
\hline & $\begin{array}{l}\text { Ecole Supérieure du Commerce Extérieur (Francia) } \\
\text { Adquirida en } 2001 \text {. Más del } 90 \% \text { de la propiedad. } \\
\text { Aproximadamente } 1.000 \text { estudiantes. }\end{array}$ \\
\hline & $\begin{array}{l}\text { Glion Institute of Higher Education (Suiza) } \\
\text { Adquirido en 2002. Propiedad completa. } \\
\text { Aproximadamente } 1.200 \text { estudiantes. }\end{array}$ \\
\hline & $\begin{array}{l}\text { Academ ia de Idiom as y Estudios Profesionales (Chile) } \\
\text { Adquirida en 2003. Propiedad completa. } \\
\text { Aproximadamente } 5.000 \text { estudiantes. }\end{array}$ \\
\hline & $\begin{array}{l}\text { Universidad Andrés Bello (Chile) } \\
\text { Adquirida en } 2003 . \text { Propiedad parcial. } \\
\text { Aproximadamente } 16 \text { mil estudiantes. }\end{array}$ \\
\hline & $\begin{array}{l}\text { Universidad Interamericana (Costa Rica y Panamá) } \\
\text { Adquirida en 2003. Propiedad completa. } \\
\text { Aproximadamente } 2.800 \text { estudiantes en Panamá y } \\
3.000 \text { en Costa Rica. }\end{array}$ \\
\hline
\end{tabular}

Fuentes: Página web de Laureate Inc. (www.laureateuniversities.com). Páginas web de las instituciones. Estados financieros de Sylvan Learning Systems Inc. actualizados en 17 de mayo de 2004. Nota: Los datos de matrícula y el porcentaje de propiedad de Sylvan sobre las instituciones pueden no ser exactos en todos los casos. 
La educación superior transnacional en México...

sando opciones de "doble titulación" mediante alianzas con otras instituciones de la red o fuera de ella.

Un aspecto importante en el desarrollo de la red universitaria, ha sido la búsqueda de formas de integración de orden horizontal (relaciones entre las instituciones educativas que forman la red) y vertical, es decir relaciones entre las instituciones y otras empresas $u$ organizaciones relacionadas con el perfil académico de las mismas. El primer supuesto se cumple a través de convenios que establecen opciones para que los estudiantes puedan realizar estancias o estudios en las diferentes unidades académicas que integran la red, mientras que el segundo implica el desarrollo de relaciones empresariales con organizaciones de la iniciativa privada, con el gobierno y con el sector social. Así, por ejemplo, las escuelas de hotelería de Europa mantienen convenios con la industria de hospedaje, las Universidades Andrés Bello (Chile) y Europea de Madrid (España) cuentan con hospitales y clínicas. En la mayoría de las escuelas y universidades se han establecido unidades para la enseñanza de inglés y para el desarrollo de software educativo, que en general se aprovechan para impulsar el modelo Sylvan en la institución respectiva pero también ofrecen servicio al público.

En tanto que grupo empresarial, el consorcio Sylvan está principalmente orientado a la consecución de ganancias, cualquier otro propósito depende de este primer objetivo. En los últimos años han sostenido una tendencia de crecimiento de sus ventas en el rango entre 20 y 25 por ciento. ${ }^{11}$ La perspectiva financiera del consorcio consiste en lograr en los próximos años un promedio de ventas anuales (sólo en la división de educación superior) de más de 600 millones de dólares al año, con una ganancia neta anual en torno a los 100 millones de dólares. Además se plantean conquistar el objetivo de mediano plazo (antes de 2010) de adquirir nuevas instituciones hasta alcanzar una matrícula total de $200 \mathrm{mil}$ estudiantes y un promedio de ventas cercano a mil millones de dólares al año, ${ }^{12}$ cifras que colocan al consorcio como el principal proveedor de educación superior transnacional en el mundo.

Por último, conviene mencionar que una nota distintiva de las instituciones afiliadas a la red es la preocupación por participar en las iniciativas de acreditación académica de cada país, así como en las fórmulas de acreditación internacional cuando es el caso. En general, el consorcio ha preferido la compra o asociación con instituciones que cuentan pre- 


\section{Tabla 4}

Matrícula total de las instituciones de educación superior incluidas en la Red Internacional de Universidades Sylvan (Laureate) 1998-2004

\begin{tabular}{|c|c|c|c|c|c|c|}
\hline 1998 & 1999 & 2000 & 2001 & 2002 & 2003 & $2004 /^{\mathrm{e}}$ \\
\hline 4.500 & 13.000 & 53.000 & 63.000 & 75.000 & 117.000 & 130.000 \\
\hline
\end{tabular}

Fuente: Página web de Laureate Inc. (www.laureateuniversities.com).

\section{Tabla 5}

Ingresos (revenues) consolidados de Sylvan Learning Systems Inc. (1996-2004)

En millones de US dólares

\begin{tabular}{|c|c|c|c|c|c|c|c|c|}
\hline 1996 & 1997 & 1998 & 1999 & 2000 & 2001 & 2002 & 2003 & $2004^{*}$ \\
\hline 104.1 & 118.1 & 178.8 & 277.0 & 316.5 & 484.8 & 604.0 & 472.8 & 132.2 \\
\hline
\end{tabular}

Fuente: Sylvan Learning Inc. Estados financieros consolidados (reportes anuales).

* Los datos 2004 se refieren al primer cuatrimestre del año.

viamente con acreditación formal de sus programas académicos, o bien ha impulsado la concurrencia en las instancias respectivas en aquellos casos en que no había cumplido previamente con el requisito de acreditación. Por ello, en la agenda estratégica de Sylvan para los próximos años se plantea la necesidad de cabildear para lograr una mayor apertura internacional del mercado de educación superior y, al mismo tiempo, apoyar la eliminación de regulaciones gubernamentales de acceso a mercados, control de la oferta educativa y control de los mecanismos de acreditación. También se propone incidir en acciones que favorezcan el propósito de participar en la distribución de fondos y contratos públicos para investigación, desarrollo e innovación, así como en la distribución de fondos públicos para becas y créditos educativos.

La actuación de Sylvan no ha estado exenta de críticas en medios académicos, políticos y financieros. Algunas críticas cuestionan posibles efectos de distorsión cultural (por ejemplo, Altbach, 2004); otra vertiente critica posibles efectos negativos de mercado en el segmento privado. Esta crítica es particularmente incisiva en medios como el chileno o el mexicano en que las universidades asociadas al consorcio ocupan, respectivamente, el primero y segundo lugar en términos de la pobla- 
La educación superior transnacional en México...

\section{Agenda estratégica de Sylvan International Universities}

- No discriminación por status legal (equidad legal para las instituciones públicas y privadas).

- Calidad académica como criterio primario que deben usar los gobiernos en la toma de decisiones.

- Igualdad de oportunidades para que todas las universidades puedan competir por fondos de investigación, desarrollo e innovación.

- Menos obstáculos regulatorios de los gobiernos y más acreditación por cuerpos independientes.

Fuente: Presentación del grupo Sylvan en el OECD/Norway Forum on Trade in Educational Services. Trondheim, 4 de noviembre de 2003.

ción universitaria incorporada al segmento privado. Por último, están los señalamientos críticos que apuntan a la necesidad de mejorar los medios de supervisión de calidad por parte de entidades gubernamentales o civiles (González, 2003; Rama, 2003), así como una mayor visibilidad pública de las propuestas (Rodríguez Gómez, 2003) para evitar que la presencia extranjera en ámbitos locales de educación superior implique deterioros cualitativos de la oferta.

\section{b) Sylvan en México: la Universidad del Valle de México}

La presencia del consorcio Sylvan en México se debe, como se indicó anteriormente, a la asociación comercial con la Universidad del Valle de México (UVM). En el año 2000 el consorcio concretó la adquisición del 80 por ciento de la propiedad de la UVM mediante una inversión de aproximadamente 50 millones de dólares, ${ }^{13}$ monto que representa la principal inversión del consorcio en una sola unidad académica hasta la fecha. Al momento de ser adquirida por el grupo Sylvan, la UVM contaba con un total de 13 campus y una población escolar de aproximadamente 35 mil estudiantes en programas de bachillerato, licenciatura y postgrado, gracias a una política de expansión y descentralización iniciada en 1976.

En el desarrollo histórico de la institución son discernibles dos fases de expansión. La primera, en los años de 1970 y de 1980, está 
representada por la apertura de planteles en la zona metropolitana de la Ciudad de México. La segunda fase se inicia en los años de 1990 y, más que un perfil de descentralización, asume la forma de un proceso de distribución territorial. Así, en 1998 se abre la sede Villahermosa (Tabasco), y en 1999 se inauguran los planteles de San Luis Potosí (San Luis Potosí) y Tuxtla Guitiérrez (Chiapas). El mecanismo de distribución territorial, similar al desarrollado por el Instituto Tecnológico de Estudios Superiores de Monterrey (ITESM), prosigue y se intensifica en el período posterior a la inversión de Sylvan. En 2000 con el plantel Texcoco, en 2001 la sede Aguascalientes (Aguascalientes), en 2002 Puebla (Puebla), en 2003 Toluca (Estado de México) y en 2004 los planteles de Guadalajara y Saltillo (Coahuila). Cabe hacer notar que los nuevos planteles del período 2001-2004 han sido instalados en ciudades capitales de los estados, lo que indica una estrategia de mercado renovada.

Naturalmente la expansión del número de planteles implicó un considerable aumento en el número de estudiantes matriculados. Según datos de la UVM, en la actualidad (primer semestre de 2004) están inscritos en la institución un total de 42 mil estudiantes, lo que comprende los niveles de bachillerato, licenciatura y postgrado en sus distintas modalidades y opciones; de esa cifra, aproximadamente 30 mil son estudiantes de licenciatura. ${ }^{14}$ Tanto el número de planteles con los que cuenta como su volumen de estudiantes implican que la UVM es la segunda universidad privada del país en términos cuantitativos. ${ }^{15}$ No obstante, debe tenerse en cuenta que en México la oferta de educación superior privada está sumamente pulverizada, de manera que la población de licenciatura de la UVM representa acaso un cinco por ciento del conjunto.

El monto de colegiaturas pagado por los estudiantes es muy variable entre planteles y niveles escolares, sin embargo la cifra de 40 mil pesos anuales por alumno representa una estimación aceptable (véase Tabla 6). Tal promedio sitúa a la institución en un rango intermedio ${ }^{16}$ dentro del grupo de universidades y escuelas superiores privadas del país y hace notar una estrategia de mercado bien definida en este aspecto.

Al considerar, por otra parte, el total de población escolar matriculada en la UVM, así como el monto anual de las colegiaturas, es claro que la aportación de la institución dentro de la suma de ingresos del grupo Sylvan es significativa: al menos una tercera parte de las ventas totales del consorcio (aproximadamente 160 millones de dólares por 
La educación superior transnacional en México...

\section{Tabla 6}

Planteles, años de creación y datos de matrícula escolar de la Universidad del Valle de México

\begin{tabular}{|l|c|c|c|c|}
\hline \multirow{2}{*}{ Plantel } & \multirow{2}{*}{$\begin{array}{c}\text { Año de } \\
\text { creación }\end{array}$} & $\begin{array}{c}\text { Picenciatura } \\
\text { (2002) }\end{array}$ & $\begin{array}{c}\text { Postgrado } \\
(2002)\end{array}$ & $\begin{array}{c}\text { Colegiatura } \\
\text { anualizada } \\
\text { por alumno } \\
\text { Pesos 2004 }\end{array}$ \\
\hline San Rafael (DF) & 1960 & 4.760 & 217 & 42,850 \\
\hline Roma (DF) & 1976 & n.d. & n.d. & 29,000 \\
\hline San Ángel (DF) & 1977 & 976 & 254 & 48,380 \\
\hline Tlalpan (DF) & 1979 & 5.108 & 180 & 49,260 \\
\hline Lomas Verdes (Estado de México) & 1982 & 6.047 & 730 & 55,410 \\
\hline Juriquilla (Querétaro) & 1988 & 2.410 & 89 & 54,160 \\
\hline Insurgentes Norte (DF) & 1989 & 673 & 146 & 38,160 \\
\hline Chapultepec (DF) & 1993 & 1.019 & 44 & 42,450 \\
\hline Lago de Guadalupe (Estado de México) & 1997 & 626 & 7 & 42,120 \\
\hline Villa Hermosa (Tabasco) & 1998 & 2.734 & 511 & 26,990 \\
\hline San Luis Potosí (SLP) & 1999 & 536 & 36 & 41,500 \\
\hline Tuxtla Gutiérrez (Chiapas) & 1999 & n.d. & n.d. & 30,590 \\
\hline Texcoco (Estado de México) & 2000 & 567 & n.d. & 34,830 \\
\hline Aguascalientes (Aguascalientes) & 2001 & 35 & 13 & 46,040 \\
\hline Puebla (Puebla) & 2002 & -- & -- & 41,480 \\
\hline Toluca (Estado de México) & 2003 & -- & -- & 41,190 \\
\hline Guadalajara & 2004 & -- & -- & 48,660 \\
\hline Saltillo & 2004 & -- & -- & 44,480 \\
\hline
\end{tabular}

Fuente: Dato de año de creación, página web de la UVM (www.uvmnet.edu/). Estadísticas de matrícula, ANUies, Anuario Estadístico, 2002.

Notas: 1 . El cuadro no incluye población de estudiantes de bachillerato. En la página web de la UVM se establece la existencia de "más de 42 mil estudiantes" en total (2004).

2. La colegiatura anualizada se estimó multiplicando por dos el costo del semestre de licenciatura sin idioma. En el caso del plantel Roma (DF) se consideró el promedio de cuotas de las diferentes opciones de bachillerato que se ofrecen.

3. El campus Roma (DF) sólo incluye estudiantes de bachillerato 
año) proceden de las colegiaturas y otros ingresos de la unidad mexicana, lo que permite suponer que la empresa continuará apoyando los proyectos de expansión de la institución.

Además de los tendencias de crecimiento y financiamiento de la universidad, es claramente observable una tendencia de adecuación de la oferta educativa a las expectativas cualitativas de la población potencialmente demandante. Por un lado, la institución ha combinado su oferta tradicional con nuevas opciones y especialidades, principalmente en torno a tecnologías informáticas, así como con ofertas específicas para estudios profesionales de tiempo parcial, es decir, las denominadas "licenciaturas ejecutivas". Además se subraya la ventaja comparativa del acceso a la red universitaria Sylvan y la enseñanza intensiva de una segunda lengua, así como los convenios de la UVM con empleadores para la colocación de egresados.

En la misma tesitura, y con clara referencia al proyecto estratégico de Sylvan, la UVM ha mostrado disposición para participar en los procedimientos de evaluación y acreditación externa, así como interés por incluirse en las principales asociaciones universitarias del país. A la fecha cuenta con nueve programas de licenciatura acreditados por las agencias reconocidas por el Consejo para la Acreditación de la Educación Superior (en áreas de contaduría, administración y psicología) ${ }^{17}$ y ha solicitado la evaluación de otros de sus programas.

En complemento de la línea de aseguramiento de calidad que procede a través de la acreditación de programas, la UVM ha desarrollado un plan de reforma denominado Modelo Educativo Siglo XXI (MES $\mathrm{XXI}$ ), el cual prevé las siguientes acciones de transformación académica: a) Organización departamental; b) Currículum flexible; c) Asesorías personalizadas; d) Metodología educativa basada en el aprendizaje activo y significativo; e) Formación docente bajo los principios del MES XXI; f) Evaluación integral del aprendizaje. ${ }^{18}$

Además de los procesos de transformación académica indicados, son destacables las líneas de integración vertical promovidas por la UVM, particularmente las centradas en actividades empresariales. En primer lugar, su participación en la sociedad NORMEX, en colaboración con la Cámara Nacional de la Industria de la Transformación (CANACINTRA) y los Laboratorios Nacionales de Fomento Industrial (LANFI), que ofrece servicios de metrología, normalización y verificación a la industria. En segun- 
La educación superior transnacional en México...

\section{Tabla 7}

Programas académicos de la Universidad del Valle de México con acreditación vigente por organismos reconocidos por el Consejo Nacional de Acreditación de la

Educación Superior A.C. (COPAES)

\begin{tabular}{|l|c|c|c|}
\hline Programa & Unidad académica & Vigencia & Organismo acreditador \\
\hline Contaduría Pública & San Rafael & Junio 2005 & CACECA \\
\hline Administración de Empresas & San Rafael & Junio 2005 & CACECA \\
\hline Contaduría Pública & Tlalpan & Junio 2005 & CACECA \\
\hline Administración de Empresas & Tlalpan & Junio 2005 & CACECA \\
\hline Psicología & Chapultepec & Noviembre 2004 & CNEIP \\
\hline Psicología & San Rafael & Noviembre 2004 & CNEIP \\
\hline Contaduría Pública & Lomas Verdes & Junio 2005 & CACECA \\
\hline Administración de Empresas & Lomas Verdes & Junio 2005 & CACECA \\
\hline Contaduría Pública & Querétaro & Junio 2005 & CACECA \\
\hline Administración de Empresas & Querétaro & Junio 2005 & CACECA \\
\hline Psicología & Querétaro & Noviembre 2004 & CNEIP \\
\hline
\end{tabular}

Fuente: Consulta SESIC/COPAES (14 de mayo de 2004)

Nota: Siglas: CAECA: Consejo de Acreditación en la Enseñanza de la Contaduría y la Administración A.C., CNEIP: Consejo Nacional para la Enseñanza e Investigación en Psicología A.C.

do lugar, la creación (en 2000) del Centro de Alto Desarrollo y Educación en Línea (CADEL), que ofrece servicios de capacitación y actualización mediante el diseño de cursos y plataformas de instrucción para grupos específicos, incluyendo sus propio personal docente y directivo.

\section{Corolarios}

Las posibilidades de acceso de capital foráneo para inversiones en el campo de la educación superior privada en México queda claramente ilustrado con el ejemplo Sylvan-UVM. Este caso resulta ilustrativo de varias posibles implicaciones de la apertura comercial en el sector. Entre ellas indicamos las siguientes:

a) En primer lugar, se demuestra que las limitaciones previstas en la normativa para inversiones extranjeras y los tratados de

1062 Educ. Soc., Campinas, vol. 25, n. 88, p. 1044-1068, Especial - Out. 2004

Disponível em <http://www.cedes.unicamp.br> 
libre comercio no han sido obstáculo, cuando menos no en el caso estudiado, para lograr asociaciones comerciales con IED por encima del criterio de 49 por ciento de participación del capital foráneo. Asimismo, queda claro que la adquisición de organizaciones con autorización previa para impartir programas de educación superior elimina la obligación prevista en la Ley General de Educación de tramitar nuevas autorizaciones sujetándose a un procedimiento de evaluación formal.

b) En segundo lugar, es evidente que el inversionista extranjero, al menos en el caso analizado, tiende a aprovechar y profundizar las ventajas comparativas del socio nacional. Asimismo, que buscará adecuar la oferta educativa a estándares de calidad de validez internacional, particularmente a través de la acreditación de programas y mediante el impulso de medidas de control y aseguramiento de calidad convencionales.

c) En tercer lugar, según se aprecia en el caso, la IED en instituciones privadas no encuentra un reflejo inmediato en el plano curricular sino, más bien, en procedimientos de organización, mercadeo, relaciones interinstitucionales, vinculación y relaciones con el gobierno.

d) En cuarto lugar, los objetivos empresariales de capitalización, ganancia y rendimiento hacen previsible que, una vez instalada la inversión extranjera, busque extenderse a partir de la propia organización receptora. En éste sentido, se puede anticipar que la inversión seguirá una dinámica de oferta-demanda y de inversión-capitalización apoyada por el volumen de capital de la firma central. Es también previsible que inversores locales (dueños y socios de otras universidades privadas) perciban como amenazante tal comportamiento expansivo a medida que éste se profundice.

e) No es irrelevante el hecho de que el inversor extranjero consolide sus indicadores de ventas y ganancias en la divisa del país de origen de la inversión. Esto quiere decir que escenarios de apreciación o depreciación de la moneda nacional, y otras variaciones de los factores de producción, pueden afectar el comportamiento de la inversión a mediano plazo, es de- 
La educación superior transnacional en México...

cir, modular el ritmo de expansión y fijar parámetros para el cobro de cuotas y el pago de salarios en la sede nacional.

f) Por último, es previsible que el ejemplo de Sylvan-uvm abra la puerta a nuevas inversiones y asociaciones de tipo similar. Por lo pronto, el grupo Apollo International Inc. ya abrió una oficina en México (el Grupo Educativo Apollo Internacional) mediante la cual se podría seguir una ruta similar a la establecida entre Apollo y el grupo Pitágoras brasileño.

Conviene comentar al término de esta presentación que la presencia de inversión extranjera directa en educación superior es sólo un aspecto, entre varios otros, que ejemplifica posibles implicaciones de los procesos y reglas de liberalización comercial en curso para los sistemas universitarios y los sistemas de investigación científica y tecnológica en países como México, cuyas condiciones son asimilables, con las especificidades de cada realidad nacional, a las de otros países de la región latinoamericana.

\section{Recebido e aprovado em setembro de 2004.}

\section{Notas}

1. En orden cronológico: Costa Rica, 1995; Grupo de los Tres (Colombia, Venezuela), 1995; Bolivia, 1995; Nicaragua, 1998; Chile, 1999; Unión Europea, 2000; Israel, 2000; Triángulo del Norte (El Salvador, Guatemala y Honduras), 2001; Asociación Europea de Libre Comercio (Islandia, Liechtenstein, Noruega y Suiza), 2001. Además está pendiente un tratado con Uruguay, hay negociaciones formales en curso con Japón y conversaciones para la eventual firma de un acuerdo con MERCOSUR.

2. Belice, Costa Rica, El Salvador, Guatemala, Honduras, Nicaragua, Panamá y los nueve estados del sur-sureste de México: Campeche, Chiapas, Guerrero, Oaxaca, Puebla, Quintana Roo, Tabasco, Veracruz y Yucatán.

3. A saber: Desarrollo humano, Desarrollo sostenible, Evaluación ambiental y social de los proyectos del ppr, Facilitación del intercambio comercial, Información, consulta y participación, Integración de los servicios de telecomunicaciones, Interconexión energética, Prevención y mitigación de desastres, Transporte, Turismo. Posteriormente se añadieron los rubros de Desarrollo rural y Componente indígena.

4. Los documentos que expresan las iniciativas de Estados Unidos, Australia, Nueva Zelanda y Japón pueden ser consultadas en el sitio web del GATS: <www.wto.org/english/tratop_e/ serv_e/serv_e.htm>.

5. Véase en: <www.aucc.ca/_pdf/english/statements/2001/gats_10_25_e.pdf>. 
6. Véase: SEP, "Acuerdo número 279 por el que se establecen los trámites y procedimientos relacionados con el Reconocimiento de Validez Oficial de Estudios de tipo superior". México, Diario Oficial de la Federación, 10 de julio de 2000.

7. Véase: SEP, "Acuerdo 286 por el que se establecen los lineamientos que determinan las normas y criterios generales, a que se ajustará la revalidación de estudios realizados en el extranjero y la equivalencia de estudios, así como los procedimientos por medio de los cuales se acreditarán conocimientos correspondientes a niveles educativos o grados escolares adquiridos en forma autodidacta, a través de la experiencia laboral o con base en el régimen de certificación referido a la formación para el trabajo". México, Diario Oficial de la Federación, 30 de octubre de 2000.

8. Véase: SEP, "Acuerdo 328 por el que se modifica el diverso 286 (...)". México, Diario Oficial de la Federación, 30 de julio de 2003.

9. La documentación sobre el COPAES A.C. puede verse en la página web de la Subsecretaría de Educación Superior e Investigación Científica de la SEP: <www.sesic.sep.gob.mx>.

10. Además de las instituciones citadas, el consorcio Sylvan instaló en 2003 el South Asia International Institute en la India. Aunque se abrieron inscripciones y se matricularon unos 500 estudiantes, el Instituto tuvo que suspender operaciones en el primer semestre de 2004 debido a nuevas regulaciones gubernamentales sobre universidades privadas. Los ejecutivos del consorcio mencionaron como factor del cierre la presencia de "condiciones desfavorables" por parte de la India’s Universities Grants Commission. Véase The Observatory on Borderless Higher Education, Breaking News, enero de 2004.

11. Entrevista con Douglas L. Becker (ejecutivo de Sylvan Learning Systems), Baltimore Sun, 13 de agosto de 2001.

12. Sylvan Learning Systems Inc. Annual Report 2002. Disponible en: <http://media.corporateir.net/media_files/irol/91/91846/reports/2002AnnualReport.pdf>.

13. El precio de compra proviene de una asignación de activos de US\$73.0 millones de dólares, menos US $\$ 23.6$ millones de dólares del pasivo recogido por la operación.

14. Según el registro estadístico de AnUies, la matrícula de licenciatura de la UVM al inicio de 2002 era de 25.566 estudiantes.

15. El ITESM, que es la institución de enseñanza superior privada de mayor tamaño en el país, cuenta con una matrícula de licenciatura superior a 50 mil estudiantes. La Universidad Tecnológica de México registra más de 30 mil estudiantes de licenciatura pero no tiene bachilleratos incorporados.

16. Por ejemplo, las universidades ITESM, Iberoamericana, La Salle, Anáhuac e ITAM cobran colegiaturas entre el doble y el triple de las establecidas en la uvM.

17. En México la acreditación de programas establece un periodo temporal acotado. En el caso de la UVM, además de los indicados en la Tabla 7, se han acreditado otros programas (odontología e ingeniería) que no han renovado aún el periodo y están fuera de vigencia.

18. Cfr. Universidad del Valle de México, Manual de inducción, edición 2002.

\section{Referencias bibliográficas}

\section{ADAM, S. Transnational Education Project: report and recommenda- tions; commissioned by Confederation of European Union Rector's}


La educación superior transnacional en México...

Conferences, 2001. Disponible en: <http://www.esib.org/BPC/docs/ Archives/COP003_transnational_education_project.pdf $>$. Acceso em: 2001 .

ALBORNOZ, M. La Universidad de las Américas y Sylvan Learning Systems Inc. In: CRUZANDO fronteras, nuevos desafíos para la educación superior. Santiago de Chile: Consejo Superior de Educación, 2003. p. 100-102. (Seminarios internacionales, v.7)

ALTBACH, P.G. The private sector in Asian higher education. International Higher Education, n. 29, 2002.

ALTBACH, P.G. Globalization and the university: myths and realities in an unequal world. Tertiary Education and Management, n. 1, 2004.

AMARAL, A.; MEEK, V.L.; LARSEN, I.M. The higher education managerial revolution? Dordrecht: Kluwer, 2003. (Higher education dynamics, v.3)

BARROW, C.; DIDOU-AUPETIT, S.; MALLEA, J. Globalization, trade liberation, and higher education in North America. Dordrecht: Kluwer, 2003. (Higher education dynamics, v.4)

BRETON, G.; LAMBERT, M. (Ed.). Universities and globalization: private linkages, public trust. París: Université Laval ; UNESCO, 2003.

CALLAN, P.M.; FINNEY, J.E. (Ed.). Public and private financing of higher education. Phoenix: Onyx; American Council on Education, 1997.

CALLAN, H. The international vision in practice: a decade of evolution. Higher Education in Europe, v. 25, n. 1, 2000.

DE WIT, H. Internationalization of higher education in the United States and Europe. Wesport: Greenwood, 2002.

DIDOU-AUPETIT, S. Sociedad del conocimiento e internacionalización de la educación superior en México. México: ANUIES, 2000.

ENDERS, J.; FULTON, O. (Ed.). Higher education in a globalising world. Dordrecht: Kluwer, 2002. (Higher education dynamics, v.4)

GARCÍA GUADILLA, C. Situación y principales dinámicas de transformación de la educación superior en América Latina. Caracas: UNESCO, 1998. 
GONZÁLEZ, L.E. Los nuevos proveedores externos de educación superior en Chile. In: CRUZANDO fronteras, nuevos desafíos para la educación superior. Santiago de Chile: Consejo Superior de Educación, 2003. p. 35-52. (Seminarios internacionales, v.7)

KNIGHT, J. Trade in higher education services: the implications of GATS. London: Observatory on Borderless Higher Education, 2002. Disponible en: <http://www.obhe.ac.uk>. Acceso en: 2002.

KNIGHT, J. Internationalization of higher education practices and priorities: 2003 IAU survey report. Czecoslovaquia Republic: UNITISK, 2003. Disponible en: <http://www.unesco.org/iau/internationalization/Internationalisation-en.pdf>. Acceso en: 2003.

LARSEN, K.; MARTIN, J.P.; MORRIS, R. Trade in educational services: trends and emerging issues. París: OECD, 2002.

LENN, M.P. La globalización de la educación superior: temas transnacionales de acceso, movilidad y calidad. In: CRUZANDO fronteras, nuevos desafios para la educación superior. Santiago de Chile: Consejo Superior de Educación, 2003. p. 22-29. (Seminarios internacionales, v.7).

NATIONAL EDUCATION ASSOCIATION (NEA). Higher education and international trade agreements: an examination to the threats and promises of globalization. Disponible en: <http://www.nea.org/he/global/intltrade.pdf>. Acceso en 2004.

NEAVE, G. Globalization: threat opportunity or both. IAU Newsletter. Disponible en: <http://www.unesco.org/iau/pdf/iaunew81.pdf>. Acceso en: mar. 2002.

PUSSER, B. Higher education, the emerging market, and the public good. In: AlbJerg, P.; Nevzer, G.S. (Ed.). The knowledge economy and postsecondary education. Washington, D.C.: National Academy, 2003. p. 105-126.

RAMA, C. La educación transnacional: el tercer shock en la educación superior en América Latina. In: CRUZANDO fronteras, nuevos desafios para la educación superior. Santiago de Chile: Consejo Superior de Educación, 2003. p. 85-96. (Seminarios internacionales, v.7)

RODRÍGUEZ GÓMEZ, R. La educación superior en el mercado: configuraciones emergentes y nuevos proveedores. In: Mollis, M. 
La educación superior transnacional en México...

(Ed.). Las universidades en América Latina: ¿reformadas o alteradas?. Buenos Aires: ClaCsO, 2003. p. 87-107.

RYAN, Y.; STEDMAN, L. The business of borderless education: update 2001. Camberra: Commonwealth of Australia, 2002.

SAUVÉ, P. Trade, education and the GATS: what's in, what's out, what's all the fuss about?; ponencia para el Forum OECD/Us on Trade in Educational Services, Washington D.C., mayo 23-24., 2002.

SCOTT, P. (Ed.). The globalization of higher education. Buckingham: Open University, 1998.

TASK FORCE ON HIGHER EDUCATION AND SOCIETY. Higher education in developing countries: peril and promis. Washington, D.C.: The World Bank, 2000.

UNESCO. Higher education in a globalized society. UNESCO Education Position Paper. Disponible en: <http://www.unesco.org/iau/pdf/ unesco_position_paper_globalization_he.pdf>. Acceso en 2003.

UVALIC-TRUMBIC, S. (Ed.). Globalization and the market in higher education, quality, accreditation and qualifications. París: UNESCO; AIU, 2002.

VAN DAMME, D. Higher education in the age of globalization: the need for a new regulatory framework for recognition, quality assurance and accreditation. Working Paper. París: UNESCO. Disponible en: <http:/ /www.unesco.org/education/studyingabroad/highlights/global_forum/ presentations/keynote_eng.doc>. Acceso en 2001.

VILLANUEVA, E. La educación transnacional y la Comisión Nacional de Evaluación y Acreditación Universitaria (CONEAU) en Argentina. In: Cruzando fronteras, nuevos desafios para la educación superior. Santiago de Chile: Consejo Superior de Educación, 2003. p. 131-142. (Seminarios internacionales, v.7).

WOODHOUSE, D. Globalisation: implications for education and for quality. Ponencia en la Conferencia de la AIIR. Rockhampton, Australia. Disponible en: <http://aair.org.au/2001Papers/Woodhouse.PDF>. Acceso en 3 sept. 2001.

WORLD BANK. Constructing knowledge societies: new challenges for tertiary education. Washington, D.C.: The World Bank, 2002. 